EUROPEAN ORGANIZATION FOR NUCLEAR RESEARCH

\title{
THE USE OF DETECTORS BASED ON IONISATION RECOMBINATION
}

IN RADIATION PROTECTION

\author{
A.H. Sullivan
}

CERN, Geneva, Switzerland

\begin{abstract}
Initial recombination of ionization in a gas depends on the distance between ions along the tracks of charged particles. This effect can be used as a basis for instruments that respond to different types of ionising radiation in about the way required by the Quality Factor-Linear Energy Transfer relation recommended by the ICRP for use in radiation protection.

Empirical instruments based on ionisation recombination, that have been used for radiation protection measurements are reviewed and relations derived from recombination theory which show that the response of such detectors can be readily predicted.

The usefulness of recombination instruments in radiation protection is discussed and the advantages and limitations assessed. It is shown that their main application will be as reference instruments against which other detectors can be calibrated. As an extension to using recombination detectors as reference instruments, the feasibility of specifying radiation quality in terms of ionisation recombination is investigated.
\end{abstract}

To be presented at the workshop for

Practical Implementation of Microdosimetric Counters

in Radiation Protection

Homburg/Saar, May 15-17, 1984

GE N E V A

1984 


\section{INTRODUCTION}

Radiation detectors based on ionisation recombination effectively measure the proportion of ions that recombine in a suitable ionisation chamber and under well defined conditions. This recombination, which is occuring along the tracks of primary and secondary charged particles, depends on the local ionisation density in the tracks and hence has some relation to the Linear Energy Transfer (LET) that specifies radiation Quality Factor (QF) for radiation protection purposes (1). The recombination method therefore offers the possibility of making an instrument that measures dose equivalent or $Q F$ of any ionising radiation independent of its LET. This would be an ideal response for a radiation protection instrument for use where the composition of the radiation field is a complicated mixture such as can be found outside the shield of high energy particle accelerators.

\section{EMPIRICAL METHODS}

The degree of ionisation recombination in a gas, as well as depending on the ion density, is also a function of the gas pressure and the strength of the electric field applied to separate the ions. Gas pressure and polarizing voltage are readily controllable parameters in an ionisation chamber and several attempts have been made to adjust them until the amount of recombination occuring with widely different radiations, is proportional to the accepted qualj.ty factor of the radiation.

The basic method for determining $Q F$ by measuring the amount of recombination, first described by zielczynski (2) is to compare the ionisation currents collected at two different applied voltages in a suitable ionisation chamber. The chamber needs to be made of tissue equivalent materials and be filled with TE gas to a pressure of at least $3 \mathrm{~kg} / \mathrm{cm}^{2}$. Typical polarization curves that have been obtained using gamma rays $(Q F=1)$ neutrons from a PuBe source $(Q F=7.5)$ and alpha particles from Radon gas introduced into the chamber $(Q F=20)$ are shown in Fig. 1. Two polarizing voltages, $v_{1}$ and $v_{2}$ are selected such that the relative difference in current measured at these two voltages, given by: 


$$
R=\left(\frac{i_{2}-i_{1}}{i_{2}}\right)
$$

has a value that is proportional to the $Q F$ of the three calibrating radiations. A linear relation between this current ratio and $Q F$ implies that the instrument can be used to determine the $Q F$ of any unknown radiation. If the upper voltage used is high enough the current $\left(i_{2}\right)$ will be proportional to absorbed dose and the dose equivalent can be determined from:

$$
D E=k i_{2}\left(\frac{i_{2}-i_{1}}{i_{2}}\right)=k\left(i_{2}-i_{1}\right)
$$

where $k$ is a constant determined by calibration with a known radiation.

This difference current will be obtained automatically if the ionisation chamber is in two sections with a common collecting electrode and the two polarjzing voltages applied with opposite sign to the two sections (3). Such a differential device is, in principle, a direct dose equivalent measuring instrument that will respond reasonably correctly to any penetrating ionizing radiation.

An alternative method of determining $Q F$ involves plotting the polarizing voltage against collected ion current over a range from $100-2000 \mathrm{~V} / \mathrm{cm}$ in a tissue equivalent ionisation chamber filled with TE gas to a pressure of $6 \mathrm{~kg} / \mathrm{cm}^{2}$ (4). When the plot is made on a $10 \mathrm{~g} \log$ scale a straight line can be reasonably fitted implying a currentvoltage relation of:

$$
i=k v^{n}
$$

The index $\mathrm{n}$ has been shown to be a linear function of the $Q F$ of calibrating radiations from gamma rays to alpha particles. Hence a measurement of the slope of a double logarithmic plot of current versus voltage in a appropriate ionisation chamber will give the $Q F$ of an unknown radiation field. 


\section{APPLICATION OF RECOMBINATION THEORY}

The empirically set up and calibrated instruments would approach being absolute measuring instruments if their performance could be predicted theoretically.

There are essentially two initial recombination theories, one applying to ionisation occuring in continuous columns of uniform jonisation (5) and the other applying to ionisation that occurs in isolated clusters ( 6 ). The realistic case of ionisation in a mixed radiation field will range between these two extreme limits. The two theories have been combined by determining the conditions under which recombination occuring in isolated clusters is equivalent to that occuring in long columns of uniform ionisation but that are randomly orjentated with respect to the electric field (7). Over a large range of operating conditions the two theories predict the same proportion of recombination when the ionisation density in the track, expressed as the number of ions per micron of unit density material, is 14 times the number of ions in an equivalent cluster. This relation implies that the average recombination occuring to ions produced in 0.07 microns of long, randomly orientated columns of constant specific ionisation is the same as that occuring in isolated clusters of the same number of jons.

This equivalence of theories enables the simpler cluster theory to be used for the calculation of the recombination in an ionization chamber. According to this theory the collected ion current $i$ is given by :

$$
i=i_{0}\left(\frac{1}{1+\mu x}\right)
$$

where $i_{0}$ is the available current and will be proportional to absorbed dose if the ionization chamber is tissue equivalent, $x$ is a function of applied voltage $(V)$ and gas pressure ( $p$ ) which for TE gas is given by :

$$
x=\frac{0.13 p^{2}}{v} \underset{p}{v}(-)
$$




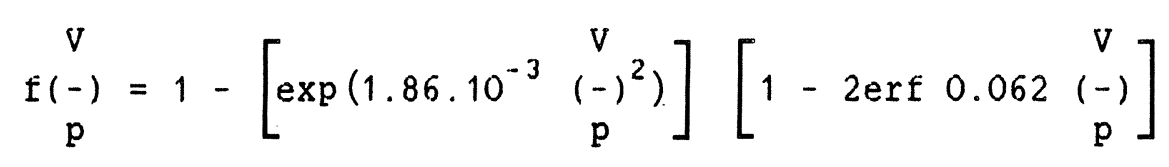

where $\mathrm{V}$ is in volts $/ \mathrm{cm}$ and $\mathrm{p}$ in $\mathrm{kg} / \mathrm{cm}^{2} . \mu$ is the number of ions per cluster (or per 0.07 microns of track) which it is supposed can be converted to LET on the basis that each ion requires an average energy deposition of $31 \mathrm{eV}$ over 0.07 microns of tissue.

Applying the above theory to a differential recombination chamber that required field strengths of $400 \mathrm{~V} / \mathrm{cm}$ and $4000 \mathrm{~V} / \mathrm{cm}$ at an absolute pressure of $3.7 \mathrm{~kg} / \mathrm{cm}^{2}$ when set up to measure dose equivalent gives the response shown in Fig. 2. Although this response does not agree exactly with the QF-LET relation it is well within what may be expected from approximations in the theory, the uncertainty in the definition of LET and in the calculation of the dose equivalent of the radiations which were used to optimize the parameters of the chamber.

\section{USE OF RECOMBINATION DETECTORS IN RADIATION PROTECTION}

As has been shown both experimentally and theoretically, recombination detectors can have a near ideal response as a function of LET for the direct measurement of dose equivalent or quality factor. However radiation protection instruments intended for measuring external radiation must have a number of properties of which an ideal response is only one. These requirements may be considered to be:

1) Adequate sensitivity.

2) Rugged and reliable.

3) Free from environmental effects.

4) Easy to read.

5) Portable.

6) Known response to pulsed radiation.

7) Known response to different radiation types and energies.

Recombination detectors so far developed do not fulfil many of these requirements. In particular the proportion of ions recombining will only be about $3 \%$ of the total current when measuring gamma rays or other low LET radiation. Hence the overall sensitivity of a differential device will be low or the chamber has to be large. This 
low sensitivity enhances the susceptibility to noise and parasitic currents due to external effects such as temperature variations and vibration. Even for relatively large chambers dose-rates below $100 \mu \mathrm{Sv} / \mathrm{hr}$ may be difficult to measure.

The use of recombination chambers in radiation protection is therefore mainly limited to measuring dose-equivalent or quality factor in relatively high radiation fields and under controlled conditions. The measured radiation fields can then be used to calibrate relatively simple but more rugged radiation protection instruments in terms of the dose equivalent of the actual radiation to be measured.

5. DEFINITION OF RADIATION QUALITY IN TERMS OF IONISATION RECOMBINATION

The application of recombination detectors as reference instruments has been extended whereby the degree of recombination serves as the practical definition of Quality Factor (8). To fulfil this function the conditions under which the recombination is measured has to be precisely defined and easily reproduced.

It has been shown that two polarizing voltages, $v_{1}$ and $v_{2}$ can be found using a suitable ionisation chamber where the measured jonisation collection efficiencies $f_{1}$ and $f_{2}$ can define radiation quality. This leads to a definition of a Recombination Index of Radiation Quality ( $R I Q$ ) which, when normalised to unity for gamma rays is given by:

$$
R I Q=\frac{\left(f_{2}-f_{1}\right)}{\left(f_{2}-f_{1}\right)} \text { gad }
$$

It is further shown that the operating voltages are correctly set in the chamber when the collection efficiency for ${ }^{60}$ co gamma rays is 0.965 at $V_{1}$ and 0.999 at $V_{2}$. These settings give the response of RIQ as a function of LET that is indicated in Fig. 2. Although this could be considered a near ideal response, the method requires determining collection efficiencies in an ionisation chamber with a high precision. 
The relation between measured currents given in equation 1 appears to offer a more easily specified definition of radiation quality. If eq. 1 is combined with the theoretical expression given by eq. 4 and normalised to unity for gamma rays, then $R$ will be given by:

$$
R=\bar{\mu}_{\gamma}^{\mu} \frac{\left(1+\mu x_{\gamma}\right)}{\left(1+\mu x_{1}\right)}
$$

where $x$ is given by eq. 5 .

This relation shows that $R$ depends on only one value of $x$ which in turn implies that only one measuring voltage needs to be specified to fix the LET dependence of $R$. The polarising voltage at which the second current is measured can be higher or lower than the specified value provided it is sufficient to avoid problems with volume recombination.

Criteria for selecting the appropriate value for $x_{1}$ could be:

1) $R=1$ when $\mathrm{L} \mathrm{ETT}=3.5 \mathrm{keV} / \mu \mathrm{m}$,

2) $R=10$ when LET $=53 \mathrm{keV} / \mu \mathrm{m}$.

To fulfill these conditions requires $x_{1}=0.005$. With this value of $\mathrm{x}$ and with $1=3.5 \mathrm{keV} / \mu \mathrm{m} \quad(\mu=7.7)$, the collection efficiency determined from eq. 4 will be $96 \%$, which should be the measured efficiency for gamma rays when the fixed chamber voltage is set correctly.

The expected LET dependence of $R$ is compared with that of $Q F$ in Fig. 3. To give an indication of how critical this response depends of the exact chamber settings, the curves corresponding to gamma collection efficiencies of 95 and $97 \%$ at the fixed polarising voltage are also plotted. As can be seen $R$ and $Q F$ reasonably correspond over the entire LET range of interest, which gives the possibility of defining radiation quality in terms of a relatively easily specified and measurable quantity for radiation protection purposes. 


\section{REFERENCES}

1. International Commission of Radiation Protection, ICRP Publication 26, Pergamon Press 1977.

2. Zielczynski, M., Recombination Method for Determination of Linear Energy Transfer of Mixed Radiation, IAEA, Proceedings of Symposium Neutron Dosimetry, Vienna 1963.

3. Zielczynski, M. and Zarnowieski, K., A Differential Recombiation Chamber IAEA-SM76/40, Proceedings of Symposium Neutron Monitoring, Vienna 1967.

4. Sullivan, A.H. and Baarli, J., An Ionisation Chamber for the Estimation of the Biological Effectiveness of Radiation, CERN Report 63-17 (1963).

5. Jaffe, G., Theorie der Ionisation in Kolonnen, Ann. der Physik $42,317(1913)$.

6. Lea, D.E., The Theory of Ionisation Measurements in Gases at High Pressures, Proc. Camb. Phil. Soc. 30, 80 (1933).

7. Sullivan, A.H., The Estimation of the Distribution of Energy Loss of Ionising Radiation from observations of the Initial Recombination of the Ions in a Gas, CERN Report 69-1 (1969).

8. Zielczynski, M., Golnik, N., Makarawicz, M., and Sullivan, A.H., Defintion of Radiation Quality by Initial Recombination of Ions, Proceedings of Seventh Symposium of Microdosimetry, Harwood Academic Publishers Ltd. Vol II, 853, Oxford 1980. 


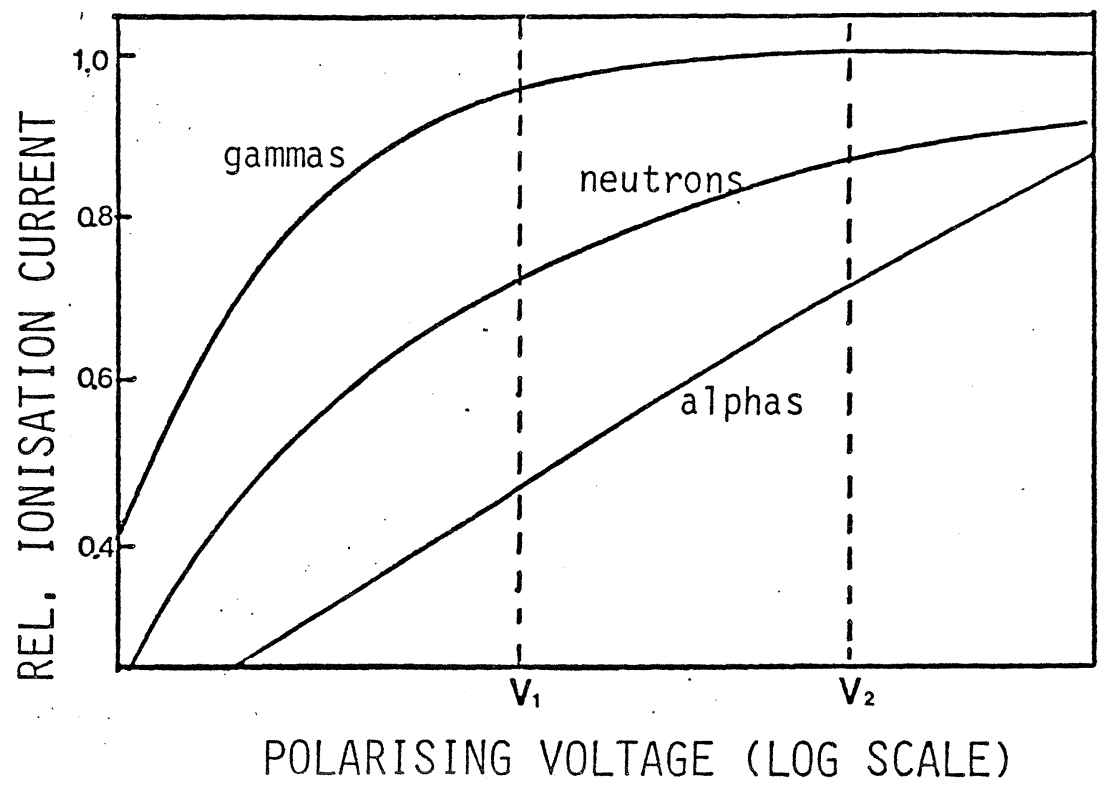

Fj.g. 1 : Variation of ionisation current in an ionisation chamber as a function of polarising voltage for gamma ray, neutron and alpha particle ionisation.

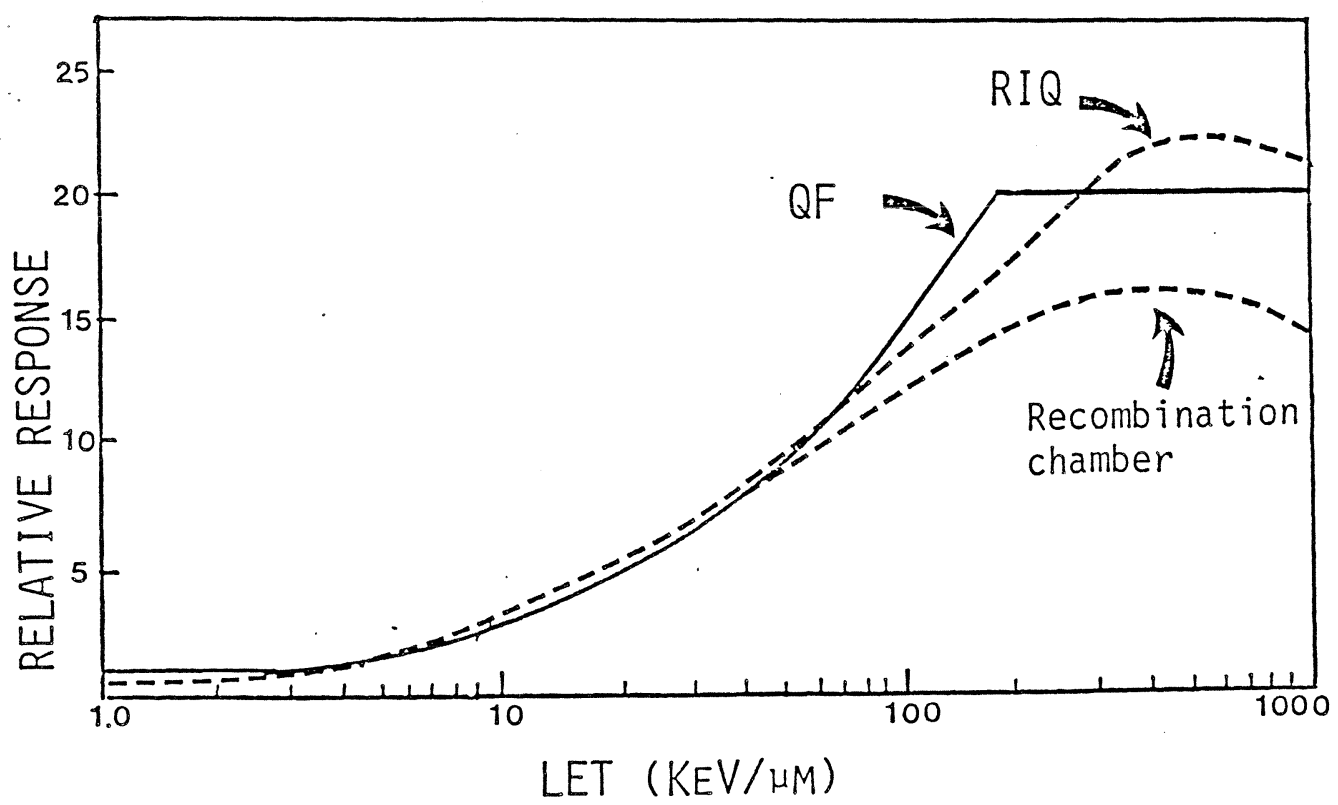

Fig. 2 : Dependence on LET of a) Quality Factor, b) Differential recombination chamber reponse and c) Recombination Index of Radiation Quality (RIQ). 


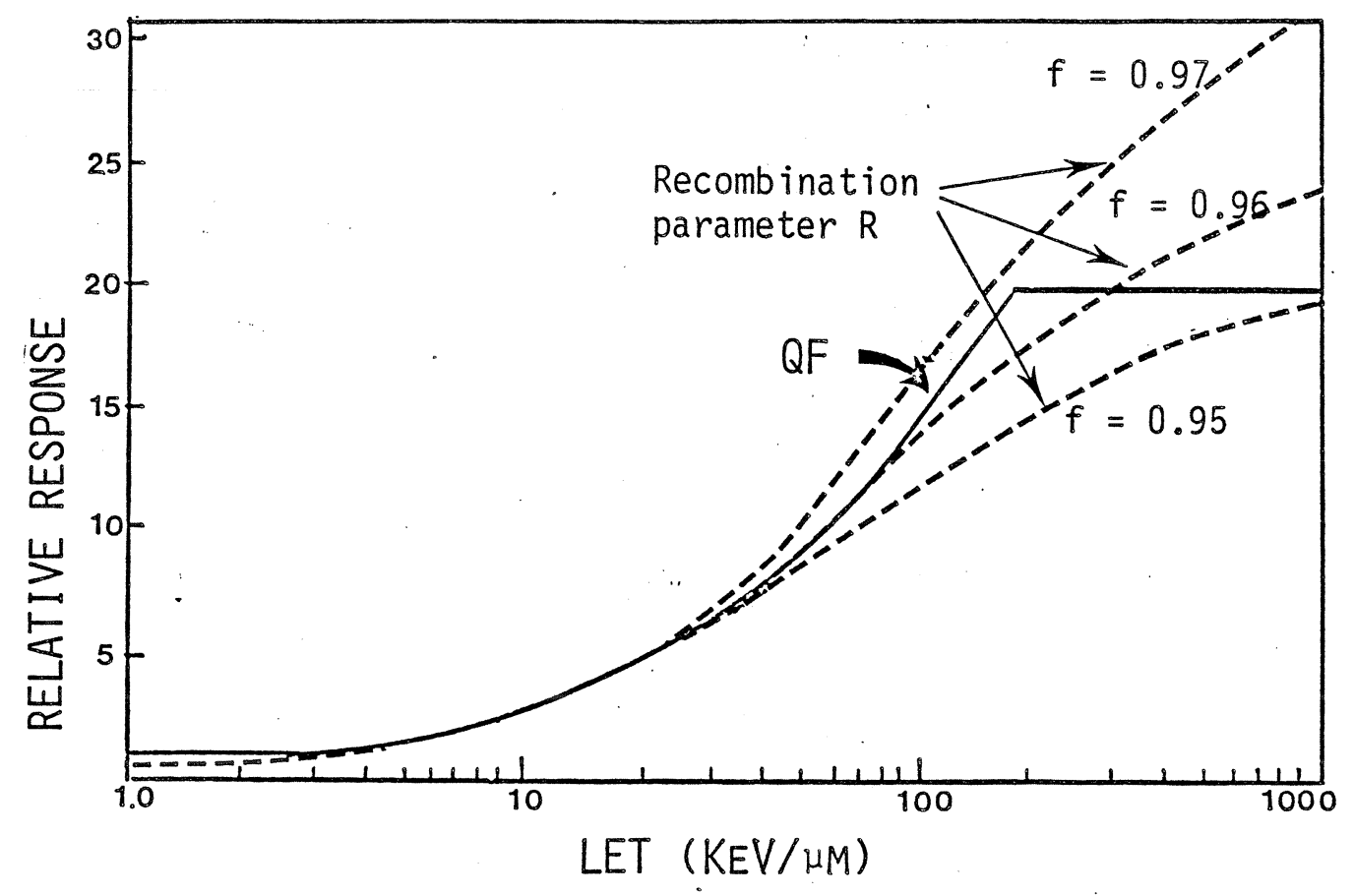

Fig. 3 : Response of the recombination parameter $R$ compared with $Q F$ as a function of LET. The response that would be obtained for a pius or minus $1 \%$ error in setting the correct collection efficiency for gamma rays is also shown for comparison. 